\title{
Handbook of Research on Driving STEM Learning With Educational Technologies
}

María-Soledad Ramírez-Montoya Tecnologico de Monterrey, Mexico

A volume in the Advances in Educational Technologies and Instructional Design (AETID) Book Series

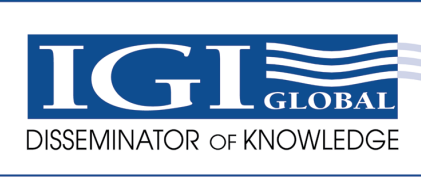

www.igi-global.com 
Published in the United States of America by

IGI Global

Information Science Reference (an imprint of IGI Global)

701 E. Chocolate Avenue

Hershey PA, USA 17033

Tel: 717-533-8845

Fax: 717-533-8661

E-mail: cust@igi-global.com

Web site: http://www.igi-global.com

Copyright (C) 2017 by IGI Global. All rights reserved. No part of this publication may be reproduced, stored or distributed in any form or by any means, electronic or mechanical, including photocopying, without written permission from the publisher. Product or company names used in this set are for identification purposes only. Inclusion of the names of the products or companies does not indicate a claim of ownership by IGI Global of the trademark or registered trademark.

Library of Congress Cataloging-in-Publication Data

Names: Ramírez Montoya, María Soledad, editor.

Title: Handbook of research on driving STEM learning with educational technologies / María Soledad Ramírez Montoya, editor.

Description: Hershey PA : Information Science Reference, [2017]

Identifiers: LCCN 2016052523| ISBN 9781522520269 (hardcover) I ISBN

9781522520276 (ebook)

Subjects: LCSH: Science--Study and teaching--United States. I

Technology--Study and teaching--United States. I Engineering--Study and teaching--United States. I Mathematics--Study and teaching--United States. I Educational technology--United States.

Classification: LCC Q181 .H14886 2017 I DDC 507.1/073--dc23

LC record available at https://lccn.loc.gov/2016052523

This book is published in the IGI Global book series Advances in Educational Technologies and Instructional Design (AETID) (ISSN: 2326-8905; eISSN: 2326-8913)

British Cataloguing in Publication Data

A Cataloguing in Publication record for this book is available from the British Library.

All work contributed to this book is new, previously-unpublished material. The views expressed in this book are those of the authors, but not necessarily of the publisher.

For electronic access to this publication, please contact: eresources@igi-global.com. 


\title{
Chapter 17 \\ A Look into Students' Interpretation of Electric Field Lines
}

\author{
Esmeralda Campos \\ Tecnologico de Monterrey, Mexico \\ Genaro Zavala \\ Tecnologico de Monterrey, Mexico \& Universidad Andres Bello, Chile
}

\begin{abstract}
On Electricity \& Magnetism (EM) courses at undergraduate level, the concept of electric field poses one of the most relevant and basic topics, along with the concept of magnetic field. Professors and students may use different diagrams as a tool to visualize the electric field, such as vectors or electric field lines. The present study aims to identify how students interpret and use electric field lines as a tool or resource to describe the electric field. Two versions of a test with open-ended questions were administered in Spanish in a private Mexican university to a random sample of students taking the EM course, and were analyzed with a qualitative approach. It was found that students do not interpret electric field lines diagrams correctly, which may lead to misconceptions. Many students based their answers on the concepts of superposition, force and repulsion.
\end{abstract}

\section{INTRODUCTION}

This chapter is focused on learning different ways in which undergrad students in introductory Electricity and Magnetism (EM) courses interpret and use electric field lines diagrams to describe the electric field at any point in space. Due to its abstract nature, the electric field is a concept that tends to be misunderstood. Several representations of the electric field may have a different effect on the correct understanding of the electric field; such as the use of vectors and the electric field lines diagram. A correct interpretation of these representations should, theoretically, lead to a better understanding of the concept of electric field, and to a correct use of concepts such as the principle of superposition, electric force and repulsion. The problem that this research tackles is to identify how students interpret electric field lines, and what effect their interpretations may have on the conceptual understanding of the electric field.

DOI: $10.4018 / 978-1-5225-2026-9 . c h 017$

Copyright $\odot$ 2017, IGI Global. Copying or distributing in print or electronic forms without written permission of IGI Global is prohibited. 


\section{In a Context}

The electric field is a concept used in physics to describe electrical interactions at a distance. The idea is that charges have an inherent ability to modify space, creating a field that interacts with other charges; it is more intense around the charge and extends to infinity, where it tends to be negligible. Unfortunately, people cannot see with the naked eye how charges modify space, although it would be very interesting. If it is not possible to see, neither to detect through the senses, then how can it be measured? The good news is that electric force can be measured, which is directly proportional to the electric field.

The electric force, according to Coulomb's law, is proportional to two interacting charges and inversely proportional to the square distance between them, as posed in Equation 1, where $q_{1}$ and $q_{2}$ are the two charges, $r$ is the distance between them and $\varepsilon_{0}$ is the permittivity of vacuum. Note that the Force $\vec{F}$ is a vector which points in the direction of $\hat{r}$, the unitary vector of the displacement vector from one charge to the other.

Equation 1: The law of Coulomb states that the electric force is proportional to two interacting charges and inversely proportional to the square distance between them.

$$
\vec{F}=\frac{1}{4 \pi \varepsilon_{0}} \frac{q_{1} q_{2}}{r^{2}} \hat{r}
$$

Imagine there is a source charge $(+Q)$ and only the force can be measured. To calculate the electric field, take a positive charge small enough such that its effect on the electric field is negligible and whose magnitude is known $(+q)$ (a test charge) and place it at the position where the field will be measured. The distance between the two charges is $\vec{r}$. Measure the force that the test charge feels and divide this quantity over the magnitude of the charge, like in Equation 2. Notice that the electric field, $\vec{E}$, is also a vector and points too in the direction of $\hat{r}$.

Equation 2: The law of Coulomb states that the electric field is proportional to the source charge and inversely proportional to the square distance between the charge and the point of interest.

$$
\vec{E}=\frac{\vec{F}}{q}=\frac{\frac{1}{4 \pi \varepsilon_{0}} \frac{Q q}{r^{2}}}{q} \hat{r}=\frac{1}{4 \pi \varepsilon_{0}} \frac{Q}{r^{2}} \hat{r}
$$

Both the electric field and the electric force have a linear and vector nature and obey the superposition principle. The principle of superposition of electric fields states that at any given point, the resulting electric field is equal to the vector sum of the electric field contributions that each source exerts on that position as if it stood by itself, as represented by Equation 3 .

Equation 3: The Superposition principle of electric fields.

$$
\vec{E}=\vec{E}_{1}+\vec{E}_{2}+\ldots+\vec{E}_{n}=\sum_{i=1}^{n} \vec{E}_{i}=\int d \vec{E}
$$


The superposition principle is a powerful tool because it allows to calculate the electric field created by any distribution of charge. By convention, the electric field in a specific location points in the direction of the force that would feel a positive test charge at that specific location. This characteristic allows to represent it in several ways, usually vectors and electric field lines. These representations can be two-dimensional or even three-dimensional, and are further explained below.

\section{Vector Diagram}

The electric field can be represented by vectors creating a vector diagram. The main tool of this representation is the arrow, which has the properties of vectors: magnitude and direction (McDermott \& Schaffer, 2001). The length of the arrow shows the field's magnitude, and its orientation shows its direction. If source charges are included the picture is clearer, since it creates a direct link between the location of the charge and its effect on space (Gire \& Price, 2013). Figure 1 shows the vector representation of the electric field exerted by one positive charge $+q$.

Among the advantages of using a vector diagram is to visualize the field strength at different distances from the source charge, to identify patterns of symmetry, and to manipulate vectors geometrically. A disadvantage may be that, even though the electric field is present at every point in space, there is a limited number of positions where it can be represented in a single diagram. Table 1 summarizes the characteristics of the use of vectors to represent the electric field.

\section{Electric Field Lines Diagram}

The electric field lines diagram consists of a pattern of lines extending from the source charge to other charges or to infinity. To represent direction, a little arrow is always shown in the middle of the line. By convention, the arrow points in the direction in which a positive test charge would feel a force if placed in this field, as shown in Figure 2. Table 1 summarizes and compares the characteristics of vectors and electric field lines as representations of the electric field.

Figure 1. Vector representation of the electric field by one positive charge

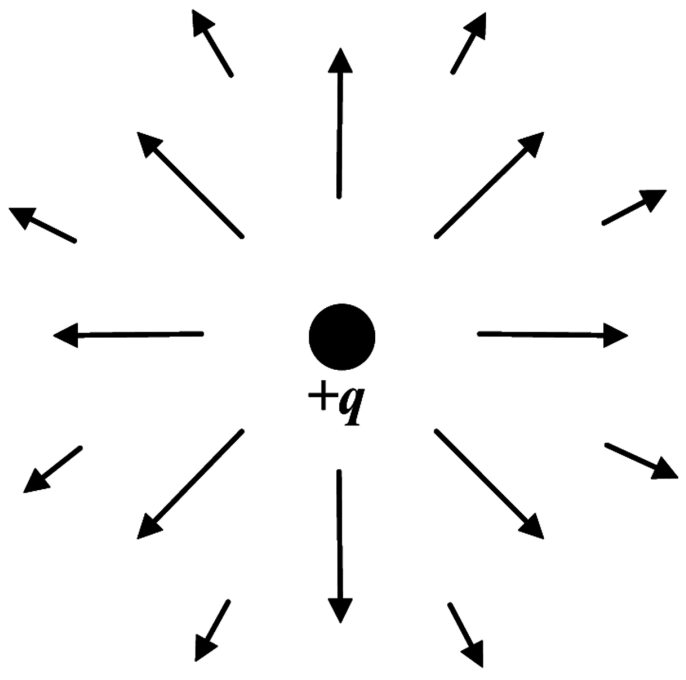


Properties of Field Lines (Serway \& Jewett, 2010)

1. The electric field vector, $\vec{E}$, is tangent to the lines at any point.

2. The number of lines per unit of length passing through a line perpendicular to the lines for a twodimensional diagram or the number of lines per unit surface passing through a surface perpendicular to the lines for a three-dimensional diagram is proportional to the electric field intensity in that region.

Rules for Drawing Electric Field Lines (Serway \& Jewett, 2010)

1. The lines begin on positive charges and end on negative charges or infinity.

2. The number of lines entering or leaving a charge is proportional to the amount of charge.

3. The field lines cannot cross each other.

The field lines diagram is a representation of the concept of the electric field as the means of action at a distance between charged objects. Faraday, who proposed the concept of electric field, imagined this field as a pattern of lines representing an invisible reality (Törnkvist, Pettersson, \& Transtromer, 1993). Instead of proposing that a charge directly affects another one at a distance, Faraday used the electric field to propose that a charge affects the space around it. As another object enters that space, it is affected by the field. Thus, the interaction occurs charge to field, and field to charge.

Similar to the vector diagram, field diagrams have some advantages and disadvantages. The main advantage is that the diagrams are very attractive. The characteristics of field diagrams are such that it is not left with a mistaken interpretation once one has understood those characteristics. However, on the down side, field diagrams are extremely difficult to construct. Students are shown the three or so more common diagrams but the rest are almost impossible to construct. As with the other type of diagram, students might think that in locations where no lines are going through, then there is no electric field.

Figure 2. Electric field line diagram for one positive charge $+q$

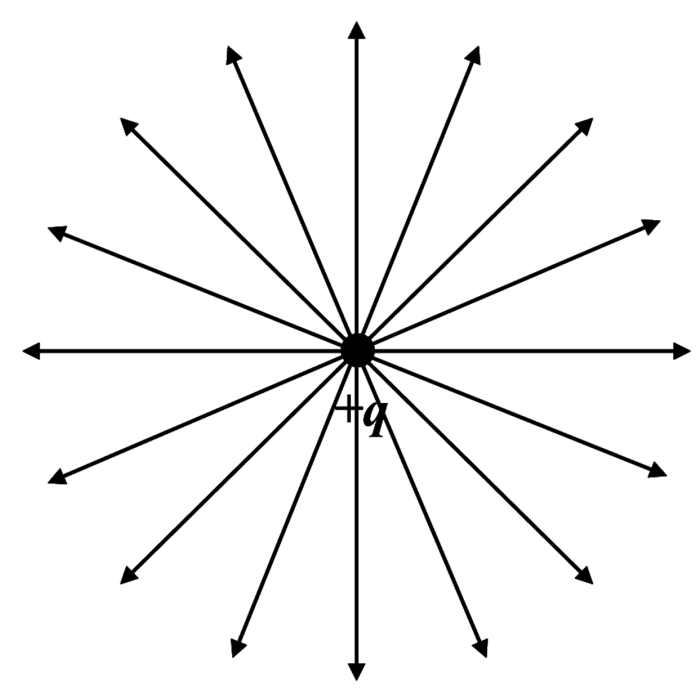


Table 1. Characteristics of the vector representation and electric field lines diagram

\begin{tabular}{|l|l|}
\hline \multicolumn{1}{|c|}{ Vector Representation } & \multicolumn{1}{c|}{ Electric Field Lines Diagram } \\
\hline $\begin{array}{l}\text { The arrow starts at the position where the electric field } \\
\text { wants to be measured. It ends defining the size of the } \\
\text { arrow. }\end{array}$ & $\begin{array}{l}\text { The lines start at the source charge, and have arrows indicating the } \\
\text { direction of the line. They leave a positive charge, and enter a negative } \\
\text { charge, or extend to infinity. }\end{array}$ \\
\hline $\begin{array}{l}\text { The size of the arrow represents the magnitude of the } \\
\text { electric field, while the orientation represents the direction } \\
\text { of the field. }\end{array}$ & $\begin{array}{l}\text { The density of field lines represents the field's magnitude, and the } \\
\text { direction of the electric field is tangent to the field line at the position } \\
\text { where it wants to be measured and in the direction of the arrow to the line. }\end{array}$ \\
\hline
\end{tabular}

\section{Problem Statement}

In previous studies performed by the authors, it was found that misinterpretations of the electric field lines diagram may result in a misapplication of the superposition principle in introductory EM students at the undergraduate level (Garza \& Zavala, 2013; Zavala, Campos \& Guisasola, 2016). This is worrying because the superposition principle is one of the most powerful tools that students have when calculating electric fields exerted by known distributions of charge. On the other hand, many textbooks for introductory EM courses present electric field lines but few of them make a clear transition between the vector representation and the field lines diagram, which results in confusion (Törnkvist, Pettersson \& Transtromer, 1993). Students need to learn how to interpret electric field lines diagrams in order to get a general idea about the electric field when the distribution of charge is unknown. After these aforementioned previous findings, there is a need to further investigate students' interpretation of the electric field lines diagram.

Figure 3 displays a diagram of electric field lines for the case of two positive charges, which has all the elements: electric field lines and source charges. As it can be seen, there is a gap between the charges leading to think that the electric field from one charge does not affect the other side. There is a possibility that the reasoning behind this is related to the presence of charges in the diagram. In previous studies, some comments like "the field lines that leave charge A do not reach to the other side, so they do not affect point P" were identified (Zavala, Campos \& Guisasola, 2016). This blocking effect may be caused by a visual disadvantage of the diagram when all of the elements are present. From this presumption, the following question arises: what would happen if students were shown a diagram of electric field lines omitting the charges that create it? Would it favor the interpretation of the diagram or would it have a negative effect?

Figure 4 displays a diagram for the same cases, but without the charges that create the field. The hypothesis is that removing the charges from the diagram will lead to a better understanding of the field as a whole, instead of thinking of it as "the field that leaves one charge".

The main objective of this study is to understand if the presence of charges in the electric field lines diagram has a negative effect on students' interpretation of the diagram, on the application of the superposition principle, and on their overall understanding of the electric field. The main question is: How do certain elements in the electric field lines diagram, specifically charges, affect students' interpretation of the diagram and the application of the principle of superposition? 
Figure 3. Electric field lines diagram for two positive charges

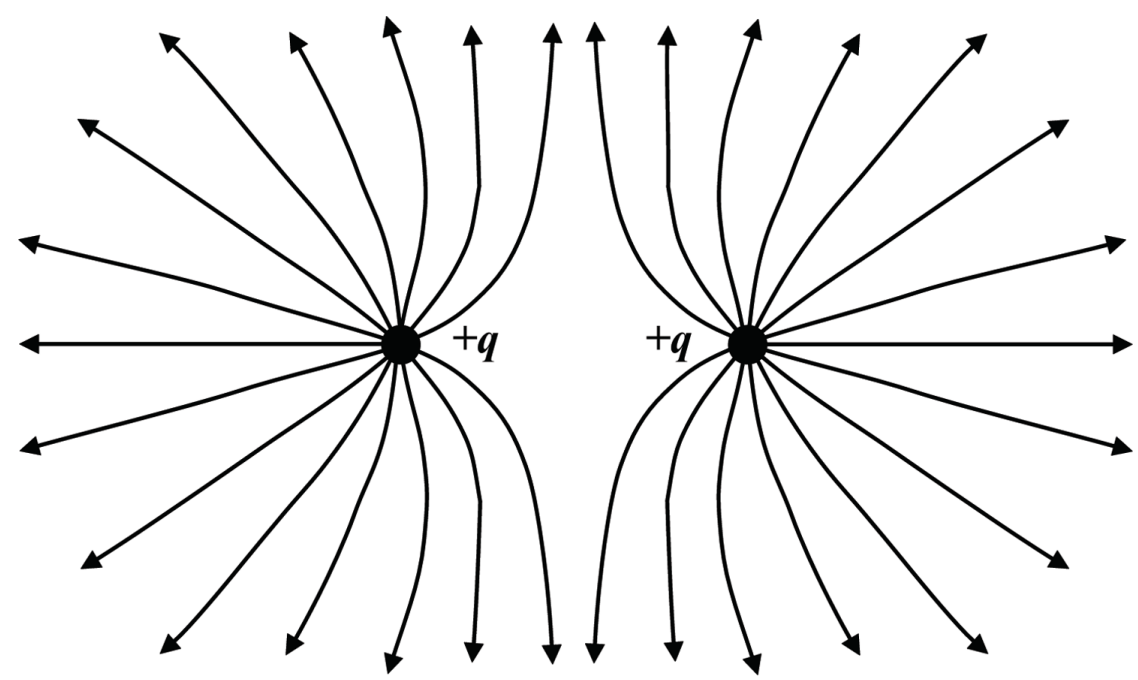

Figure 4. Electric field lines diagram for two positive charges, without the charges

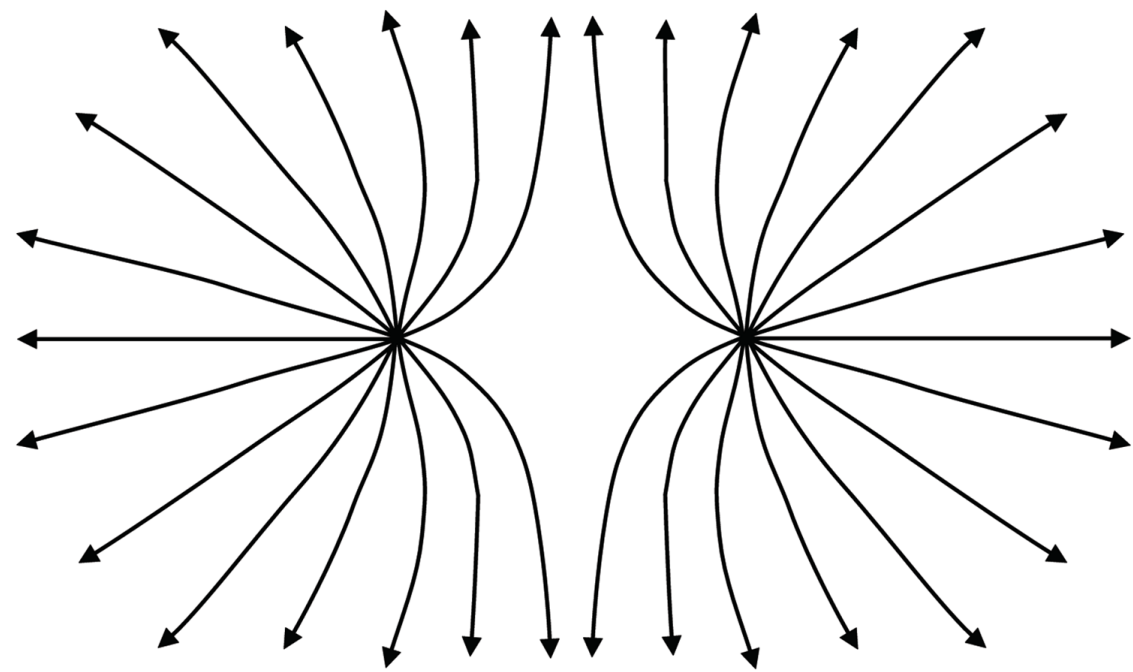

\section{LITERATURE REVIEW}

In Physics Education Research, Electricity and Magnetism has been of interest for the last decades, focusing mainly in electrostatics and circuits (McDermott, 1999). In electrostatics, much of the research has centered on electrical interactions, but the conceptual understanding of the electric field in relation to its representations remains rather unexplored. As shown in Table 2, the most relevant studies on this topic have suggested that students have conflicts with electric field lines and the application of the superposition principle for electric fields. 


\section{Two Models to Describe the Electric Field}

To describe the concept of electric field, there are two models: the action-at-a-distance model, which requires a test charge to measure electric force, and the field model, which explains the electric field as a continuous action in space (Furio \& Guisasola, 1998). Regarding the action-at-a-distance model, several studies have led to the conclusion that students tend to mix the concepts of field strength and electric force (Furio \& Guisasola, 1998; Garza \& Zavala, 2013). Furthermore, the concept of electric force is better understood by students, since they have a higher tendency to apply the superposition principle for electric force than for electric field (Garza \& Zavala, 2013).

On the other hand, the field model is usually represented by lines of force as proposed by Faraday, also known as electric field lines. The field model brings the concept of potential into the picture, but its representation by field lines has a few limitations (Furio \& Guisasola, 1998). Several studies have focused on identifying these limitations, and are further explained in the paragraphs that follow.

\section{Limitations of the Field Model Representation: Electric Field Lines}

Rainson and Viennot (1992) found that most students do not use the superposition principle and generally ignore the electric field from sources outside a Gaussian surface, when using Gauss' Law as a tool to calculate the field. Furthermore, they found that students have a linked concept of moving charges to the existence of an electric field (Rainson \& Viennot, 1992). This relationship is similar to the notion in mechanics that without an external force there is no motion or speed. Törnkvist, Pettersson and Tranströmer (1993) found that students treat electric field lines as isolated entities in space, and not as a representation of the vector nature of it. They suggested that this misconception could be derived from the misconception earlier reported by Rainson and Viennot (1992) about the relationship between the movement of charges and the presence of electric field. They recommended that to avoid the confusion that electric field lines create, there should be an effective transition from the vector representation to representation by field lines (Törnkvist, Pettersson \& Tranströmer, 1993).

Electric field line diagrams are, without doubt, visually attractive for students who are learning about electric fields, but this feature does not mean that they are useful for concept understanding. In the late 90's, Wolf, Van Hook and Weeks (1996) stated that electric field line diagrams do not work, because even when they correctly indicate the direction of the field, they fail to accurately report its magnitude. They made their case by finding that there is no possible relationship that satisfies both linear and quadratic relations between density and strength, they also note that the problems presented by electric field lines diagrams can easily be avoided by using vector representation of the electric field, although it is less aesthetic (Wolf, Van Hook \& Weeks, 1996). In agreement with Wolf, Van Hook and Weeks (1996), Chabay and Sherwood (2006) emphasize that at an introductory level there are not many problems in which the field lines are absolutely necessary, and understanding this representation requires a long investment of time; instead, vectors may allow both 2D and 3D representation without as much investment since students should be familiar with the use of vectors.

\section{Blocking Effects in Electric Field Lines}

Garza and Zavala (2010) found that students tend to define the electric field through its graphical representation, instead of using electrical interactions to argue the existence of the field; they also identified two 


\section{A Look into Students' Interpretation of Electric Field Lines}

types of blocking effects in electric field lines representation: geometrical and graphical. The geometric blocking effect is associated with the barrier that a physical object represents, while the graphic blocking effect is associated with the barrier that electric field lines, as a visual representation of an abstract concept, represent (Garza \& Zavala, 2010). Garza and Zavala (2013) noticed that field line diagrams lead to an incorrect application of the superposition principle, and students who use the vector model are more likely to correctly apply this principle; additionally, they agree with Törnkvist, Pettersson and Tranströmer (1993) in that students do not understand the concept of field lines, but treat them as if they were independent between one object and another (Garza \& Zavala, 2013).

\section{Electric Field Lines vs. Magnetic Field Lines}

It is natural to relate the electric and magnetic fields because there are a few similarities between the concepts. As a result, there is a tendency for students to apply the same concept from electric to magnetic force, thinking that a resting charge can experience a magnetic force; this misconception is known

as the "charged pole" model, and was found by Maloney (1985) and replicated by Scaife and Heckler (2007), who also introduced the question of how representation by field lines affects students' answer. They conclude that different representations cue different thinking paths, in the case of magnetic field, magnetic field lines seem to have a positive effect on students' application of the right hand rule to find the direction of magnetic force (Scaife \& Heckler, 2007). In a different study conducted by Sağlam and Millar (2006) with upper high school students about their understanding in Electromagnetism, they found that students carry the same alternative conception as the "charged pole" model reported by Maloney (1985), which implies that this alternative conception is carried from high school to university studies.

Following this line of thought, that different representations are related to different models, the question arose of how each representation of the electric field affects students' reasoning. In this case, the question is if the presence of charges in electric field lines diagrams affects students' interpretation of the electric field's representation and on the application of the superposition principle.

\section{Use of Technology to Visualize the Electric Field}

\section{Computational Visualization of Field Lines}

As stated in the introduction, one of the disadvantages of electric field lines is that they are complicated to draw. Evidence suggests that students have difficulties changing from a two-dimensional visualization to a three-dimensional one (Sağlam \& Millar, 2006). To overcome these difficulties, Sousa, García, Marinho and Mouraz (2013) implemented a visualization strategy based on research to simulate electric field lines in three dimensions for a few cases, and analyzed student concepts and perception of this new visualization. According to the authors, this visualization could help bridge the gap reported by Wolf, Van Hook andWeeks (1996) about the transition from a two-dimensional to a three-dimensional model of electric field lines. 
Table 2. Summary of several studies about Electricity and Magnetism with their respective findings

\begin{tabular}{|c|c|c|c|}
\hline Authors & Year & Aim of study & Findings \\
\hline Rainson and Viennot & 1992 & $\begin{array}{l}\text { To study students' conceptualization of } \\
\text { the electric field. }\end{array}$ & $\begin{array}{l}\text { 1. Most students do not use the superposition principle, } \\
\text { ignoring the field outside a Gaussian sphere. } \\
\text { 2. Students have a linked concept of moving charges } \\
\text { (electric current) to the existence of an electric field. }\end{array}$ \\
\hline $\begin{array}{l}\text { Törnkvist, Pettersson } \\
\text { and Tranströmer }\end{array}$ & 1993 & $\begin{array}{l}\text { To study the confusion that electric } \\
\text { field lines may have on the concept of } \\
\text { superposition. }\end{array}$ & $\begin{array}{l}\text { 1. Students treat the field lines as isolated entities in } \\
\text { the Euclidean space, and not as a representation of the } \\
\text { vector nature of it }\end{array}$ \\
\hline $\begin{array}{l}\text { Chabay and } \\
\text { Sherwood }\end{array}$ & 2006 & $\begin{array}{l}\text { To propose a different structure of an } \\
\text { EM course with a comprehensive and } \\
\text { coherent sequence }\end{array}$ & $\begin{array}{l}\text { The course is focused on the understanding of field, } \\
\text { both electric and magnetic, and uses tools such as circuit } \\
\text { analysis, which is observable, to reinforce abstract } \\
\text { concepts }\end{array}$ \\
\hline Scaife and Heckler & 2007 & $\begin{array}{l}\text { To examine the role of representation } \\
\text { on students responses to magnetic force } \\
\text { questions }\end{array}$ & $\begin{array}{l}\text { 1. Students believe in "charged" magnetic poles } \\
\text { 2. Representation format affects responses about } \\
\text { magnetic force }\end{array}$ \\
\hline Garza and Zavala & 2010 & $\begin{array}{l}\text { To understand the models that students } \\
\text { have about the electric field and electric } \\
\text { interactions and to analyze students' } \\
\text { response to different degrees of guidance. }\end{array}$ & $\begin{array}{l}\text { 1. Replacing abstract objects with real objects does not } \\
\text { affect students' reasoning. } \\
\text { 2. Guiding students helps those who were not } \\
\text { completely sure of their response, but the impact of } \\
\text { guidance is limited. } \\
\text { 3. Students tend to define the electric field through } \\
\text { its graphic representation, instead of using electrical } \\
\text { interactions to argue the existence of the field }\end{array}$ \\
\hline Garza and Zavala & 2013 & $\begin{array}{l}\text { To contrast how students understand the } \\
\text { concepts of electric field and electric } \\
\text { force }\end{array}$ & $\begin{array}{l}\text { 1. Students have a clearer picture of electric force than } \\
\text { of the electric field concept. } \\
\text { 2. Field line diagrams lead to an incorrect application of } \\
\text { the superposition principle }\end{array}$ \\
\hline $\begin{array}{l}\text { Zavala, Campos and } \\
\text { Guisasola }\end{array}$ & 2012 & $\begin{array}{l}\text { To identify the effect of each } \\
\text { representation of the electric field on the } \\
\text { application of the superposition principle }\end{array}$ & $\begin{array}{l}\text { 1. Electric field lines have a negative effect on students' } \\
\text { comprehension of the superposition principle. } \\
\text { 2. Students who used vector representation have a higher } \\
\text { tendency towards correctly applying the superposition } \\
\text { principle. }\end{array}$ \\
\hline
\end{tabular}

\section{METHOD}

\section{Research Method}

The research has a qualitative approach, since its main interest relies on the reasoning that students present, and how they interpret the electric field lines diagram. This approach is distinguished by a focus on meanings, understanding and processes, includes interviews and observation as data gathering strategies, and requires an inductive and comparative analysis (Valenzuela \& Flores, 2012). According to Merriam (2009), this research type is characterized by the construction of knowledge; the researcher tries to understand the meaning that the studied phenomenon has for the participant. The students' reasoning is crucial for the present research because it cannot be identified if students are interpreting the diagram correctly based solely on their answers. Sometimes students know the answer from previous examples, but they are not really using the diagram to justify their answers. The qualitative approach is useful in this case to analyze students' reasoning and to identify the different interpretations that students make of the diagram. 


\section{Participants}

The participants were 205 randomly selected engineering undergraduate students taking the EM course in a private Mexican University during the semester of August-December 2015. Students from this private university come from different high schools all around the country. The population is highly educated; the standards to get into the university are high, which means that students are apt to learn, but not necessarily prepared in the topic. As a matter of fact, it has been demonstrated that the levels of knowledge that these students have about Physics in general is low (Zavala \& Alarcon, 2008). This sample cannot be generalized to the whole Mexican population, but it represents highly educated students in the country.

All students had previously taken two courses of Physics, mainly focused on Mechanics and direct contact interactions, but they presumably had not been formally instructed on electric interactions at a distance, considering that this was the first course in their undergraduate studies to undertake this topic. The first topic of the EM course introduced students to the concepts of charge, electric force and electric field. The second topic deepened the study of the electric field, and introduced electric field lines as well as the superposition principle. The third topic was about Gauss' Law, which reinforced the use of electric field lines. Thus, by the end of this topic, students had all the tools they might have needed to participate in the study.

\section{Instruments}

The instrument consisted of two versions of a test with open-ended questions regarding the electric field on a particular case. This choice of instrument provides detailed answers with a sample big enough to allow generalization within the specific population. Another choice could be an interview, but it does not allow generalization, and to allow it, many people needed to be interviewed and the infrastructure available is not adequate for that.

The tests were administered in Spanish upon completion of the topic of Gauss' Law. In every case, the students were asked to explicitly post the reasoning that led them to their answer.

The two versions of the test posed the same problem and questions, but the diagrams were slightly different. There was a point charge $+q$ and a point $P$ to the right, and the students were asked to describe the electric field at the point $P$. Next, there was an additional point charge $+q$ to the left of the original and the question was whether the electric field at point $P$ increases, decreases or stays the same. Version 1 (V1) showed a regular electric field lines diagram (Figure 3), while Version 2 (V2) showed the same diagram without the charges (Figure 4). Translations of V1 and V2 are shown in the Appendix. The two versions were administered randomly among students so the results of students' answers to each test are comparable (Barniol \& Zavala, 2009). V1 was answered by 103 students and V2, by 102.

The correct answers to each question were the same on both versions, since the only difference between them was the electric field lines diagram. In question 1, the correct answer is that the electric field is directed to the right and has a magnitude of $E=\frac{1}{4 \pi \varepsilon_{0}} \frac{q}{d^{2}}$. In question 2 the correct answer is to refer to Coulomb's Law for the magnitude and that the electric field line on that point is directed to the right, the electric field is tangent to the field line, so it should be to the right. The correct answer in question 3 is that the electric field increases, and in question 4 is that it increases because the electric field lines are closer together than in the diagram in question 1. Another correct answer in V1 is that it 
increases because, applying the superposition principle, both charges generate electric field to the right and they can be added to calculate the electric field at that point. This correct answer only applies to V1 of the test, because in V2 the charges are not present in the diagram, so students who answer this can only assume that there are two charges instead of one.

\section{Methodological Strategy}

Data was collected after the application and interpreted qualitatively. The data analysis strategy was content analysis, since it enriches the collection of integral, deep and interpretative results (Perez, 1994 cited by Caceres, 2003). Similar reasoning was grouped together to fit into a category. All answers to all four questions were grouped into categories, but the most relevant to this study are the categories on questions 3 and 4. The categories for the answers in question 3 are "Increases", "Stays the same" and "Decreases". The category of "Increases" considers answers such as "Increases" and "It is greater". The category of "Stays the same" includes answers such as "Stays the same" and "It is equal". The category of "Decreases" includes answers such as "It decreases" and "It is smaller". The categories for answers in question 4 are "Superposition", "The other source affects", "Field lines are cancelled", "Forces are cancelled", "The other charge does not affect", and "Repulsion". Some answers fall into more than one category, since they combine different concepts into one long and deep reasoning. The following sections mention a few examples for each of the reasoning.

\section{Superposition}

This category groups together those answers that explicitly mention the superposition principle, for example "since the two charges affect position P, it is superposition of electric fields"; and those that imply the superposition principle by mentioning the sum of contributions, for example "because there is another charge the fields are added, in this case two vectors with the same direction". The following example satisfies both conditions: "field superposition (vector sum)".

\section{The Other Source Affects}

This category includes answers that identify that the other charge affects, like: "the other charge gives it another small impulse"; that the other field affects, for example: "because both fields affect the point", and that the other source affects: "because another source is added and it is equal to the former". In some cases, students combined this reasoning with the superposition principle, and their reasoning was identified in both categories.

\section{Field Lines are Cancelled}

This category considers answers that indicate that electric field lines are cancelled between the two charges, for example "because the new field has a field that is cancelled with the previous (in the middle)"; or that electric field lines on the left do not affect to the other side, like "the electric field lines from the distant charge do not affect on point $P$ ". 


\section{Forces are Cancelled}

This category groups those answers that mention that forces are cancelled in the middle, for example: "forces are eliminated". These answers are slightly similar to the previous category and are often combined, in which cases they were considered in both categories.

\section{The Other Charge Does Not Affect}

This category includes answers that imply that the other charge, field or source does not affect or reach the other side, "because the other charge does not affect its magnitude". Some arguments include that the contribution is negligible due to the distance "the charge that exerts the new electric field is negligible because the distance is very far away".

\section{Repulsion}

This category considers answers that include repulsion, for example "both repel each other", or "a repulsion force is added, since there are more opposing vectors".

\section{RESULTS}

The results found in the study are presented in this section. First an overview of the results is shown; then, a focus on each of the main reasoning that students have, presenting sample answers as evidence for different combinations of versions, answers and reasoning.

Questions 1 and 2 of both versions of the test serve as problem statements, guiding students into questions 3 and 4. Question 3 asks the students to answer if the electric field increases, decreases, or stays the same when placing an additional source of electric field. Question 4 asks them to explain their reasoning in detail. Overall, the results for question 3 look very similar for both versions of the test as shown in Figure 5, but there are slight differences in students' reasoning found in the answers to question 4. These differences in reasoning lead to several findings concerning the presence of charges in electric field lines, misinterpretations about the density of lines, the superposition principle, and a misconception of electric force and electric field.

\section{The Presence of Charges in the Electric Field Lines Diagram}

Overall, from the results found in the differences in reasoning in question 4, as shown in Table 3, it is evident that the application of the superposition principle is somehow affected by the presence of charges in the electric field lines diagram. The diagram with charges in V1 allows students to apply the superposition principle directly, since they might analyze each charge individually. The diagram without charges in V2 leads students towards thinking that there is more charge affecting the field, since there was an increase in this reasoning in $\mathrm{V} 2$, but it does not lead them to think about the superposition of the electric field created by each charge individually, precisely because the charges are not present in the diagram.

As seen in Table 3, 47\% of students who answered increases in V1 asserted that the additional charge affects the electric field, and therefore the magnitude of the electric field at point $P$ increases. Parallel, 
Figure 5. Comparison of the answers given by students regarding whether the electric field increases, decreases or stays the same when adding a source of electric field

Version 1: Question 3

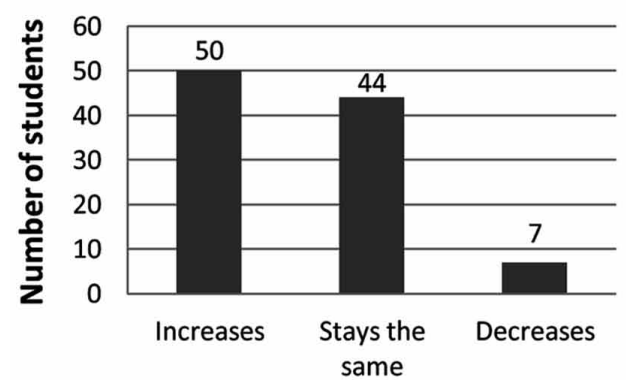

Version 2: Question 3

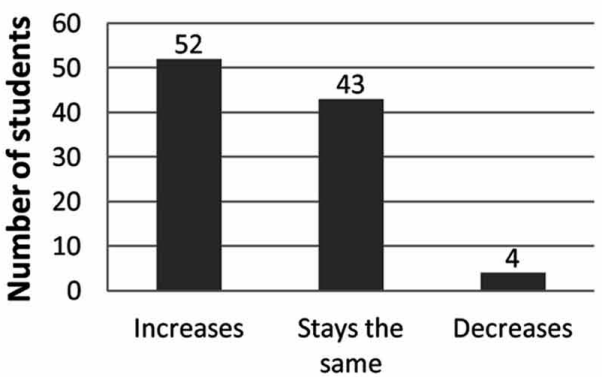

Table 3. The table organizes differences in reasoning for each version. Percentages shown correspond to students who answered each particular answer and version. For example, it reads: " $45 \%$ of students who answered Increases in V1 supported their answer on the superposition principle”.

\begin{tabular}{|l|c|c|c|c|}
\hline \multirow{2}{*}{ Reasoning } & \multicolumn{2}{c|}{ Increases } & \multicolumn{2}{c|}{ Stays the Same } \\
\cline { 2 - 5 } & V1 & V2 & V1 & V2 \\
\hline Superposition & $45 \%$ & $20 \%$ & $5 \%$ & $3 \%$ \\
\hline The other source affects & $47 \%$ & $54 \%$ & $4 \%$ & $3 \%$ \\
\hline Field lines are cancelled & $0 \%$ & $5 \%$ & $24 \%$ & $34 \%$ \\
\hline Forces are cancelled & $0 \%$ & $0 \%$ & $18 \%$ & $11 \%$ \\
\hline The other charge does not affect & $0 \%$ & $0 \%$ & $29 \%$ & $45 \%$ \\
\hline Repulsion & $8 \%$ & $20 \%$ & $20 \%$ & $5 \%$ \\
\hline
\end{tabular}

54\% of students who answered increases in V2, supported their reasoning on the additional source, but there were three types of answers identified. Some samples of the different types are illustrated in Table 4. Some students would say that the magnitude of the electric field at point $P$ increases because the new source of electric field affects, others would say that the new field affects, while others would interpret the diagram as the electric field produced by two positive charges, making the electric field stronger.

Table 4. Samples of students' answers when they mention that the additional charge or source of field affects

\begin{tabular}{|c|c|c|l|}
\hline Student & Version & Question 3 & \multicolumn{1}{c|}{ Reasoning (Question 4) } \\
\hline 1 & 1 & Increases & Because the second charge also affects at point $\mathrm{P}$. \\
\hline 2 & 1 & Increases & Because the second positive charge also exerts E at point $\mathrm{P}$. \\
\hline 3 & 2 & Increases & $\begin{array}{l}\text { The electric field increases because the field lines on the previous question seem to repel } \\
\text { each other, which indicates the presence of two charges with the same sign, in this case } \\
\text { positive, since the lines have an outward direction. Being produced by two positive charges, } \\
\text { the electric field vector on point } \mathrm{P} \text { increases slightly on direction of i. }\end{array}$ \\
\hline 4 & 2 & Increases & The second source of electric field also affects the sum of vector components in $\mathrm{x}$. \\
\hline 5 & 2 & Increases & The field on the left modifies the resulting vector in $\mathrm{P}$, it repels $\mathrm{P}$. \\
\hline
\end{tabular}




\section{Misinterpretation of Electric Field Lines: Density of Lines}

By definition, one of the main characteristics of electric field lines is that the density of lines that passes through a surface perpendicular to the line is proportional to the magnitude of the electric field in that region (Serway \& Jewett, 2010). In both versions of the test, there was an alarmingly low percentage of students who used this feature of electric field lines as a tool to support their reasoning. In V1, none of the students referred to the density of lines, while in V2, only two students vaguely mentioned the amount of lines, not the density. Both students stated that the electric field increases. Both answers are displayed on Table 5.

\section{Blocking Effect of Field Lines on the Superposition Principle}

When students answer that the electric field remains the same when adding a positive charge, they explain in their reasoning that the electric field lines that leave the charge on the left do not reach point $P$, since they are diverted by the electric field lines that leave the charge on the right. This is a blocking effect found in the electric field diagram, which may cause students to misunderstand electric field lines and not to apply the superposition principle (Zavala, Campos \& Guisasola, 2016). Some sample answers are shown in Table 6 in which students refer to the diagram to state that the electric field stays the same.

\section{Force and Repulsion}

Many students mention the concept of force when referring to the electric field, or they mention there is repulsion to justify their answers. Some sample answers are shown in Table 7. This concept might be a

Table 5. Samples of answers given by students where they include the density of field lines

\begin{tabular}{|c|c|l|}
\hline Version & Question 3 & \multicolumn{1}{c|}{ Reasoning (Question 4) } \\
\hline 2 & Increases & There are more field lines and the interaction between charges would increase the electric field \\
\hline 2 & Increases & Addition of electric field lines \\
\hline
\end{tabular}

Table 6. Samples of answers given by students where their reasoning includes a blocking effect caused by the diagram

\begin{tabular}{|c|c|c|l|}
\hline Student & Version & Question 3 & \multicolumn{1}{|c|}{ Reasoning (Question 4) } \\
\hline 1 & 1 & Stays the same & $\begin{array}{l}\text { Because the electric field from charge q1 is diverted by charge q2, therefore point P gets the } \\
\text { same field than in the previous question }\end{array}$ \\
\hline 2 & 1 & Stays the same & $\begin{array}{l}\text { The charges repel each other, so the field that would have gotten to point P is cancelled by } \\
\text { the adjacent charge. }\end{array}$ \\
\hline 3 & 2 & Stays the same & As seen in the arrows, the electric field from the second field does not reach P. \\
\hline 4 & 2 & Stays the same & $\begin{array}{l}\text { Because the force that the field on the left should exert is diverted by the force vectors from } \\
\text { the field on the right, I mean the one on the left does not affect on this case at point P. }\end{array}$ \\
\hline 5 & 2 & Increases & $\begin{array}{l}\text { Even though the electric field lines seem to cancel each other out, for the test charge you } \\
\text { need to sum all electric fields available. }\end{array}$ \\
\hline
\end{tabular}


Table 7. Samples of answers given by students where their reasoning includes the concept of repulsion

\begin{tabular}{|c|c|c|l|}
\hline Student & Version & Question 3 & \multicolumn{1}{c|}{ Reasoning (Question 4) } \\
\hline 1 & 1 & Increases & The electric field increases because there are two charges that repel each other. \\
\hline 2 & 1 & Stays the same & Because they only repel each other on the left side, the right side stays the same. \\
\hline 3 & 2 & Increases & Because the other source repels even more, and that increases the magnitude. \\
\hline 4 & 2 & Stays the same & $\begin{array}{l}\text { The vector does not affect, the magnitude and direction is constant because when the } \\
\text { vectors from the charges repel each other they do not affect to the ones that are not near. }\end{array}$ \\
\hline
\end{tabular}

double-edged sword. From what was found on the results, in V1, students have a higher tendency to use the concept of repulsion to justify that the electric field stays the same, while in V2, they have a higher tendency to justify that the electric field increases. At this point, the results obtained are very variable and there is no complete understanding of this effect with the available information.

\section{DISCUSSION}

The implications of the results found in the study are further discussed in this section. First a comparison to results found in a previous study is discussed to confirm the validity of these findings. Then, the main implications that the presence of charges may have on the application of the superposition principle are presented. About the concept of repulsion, the study is contrasted with previous studies found in the literature, the implications of these findings are discussed. Finally, a special case found in the results and its implications on more complicated cases are analyzed.

\section{Blocking Effect Caused by Electric Field Lines}

As found by Garza and Zavala (2013), students interpret electric field lines as independent from one object to another. Some of the characteristics of electric field lines are that they never cross and that they leave or enter charges depending on their sign (Serway \& Jewett, 2010). For certain distributions electric field lines that leave one charge look diverted by other field lines precisely because they do not cross; such is the case of the electric field caused by two positive charges. This characteristic of electric field lines combined with the notion that field lines are independent from one object to another may create a blocking effect on students' application of the superposition principle for electric fields.

The deviation of field lines does not mean that the electric field that "leaves" one charge does not "reach" the other side; the superposition principle states that to know the electric field at a specific point, you can analyze the electric field created by each source as if it were by itself and perform a vector addition of the contributions. In a previous unpublished study, this blocking effect was found and analyzed in a deeper sense (Zavala, Campos \& Guisasola, 2016). V1 is based on the version with electric field lines from the preliminary study. The results found in the previous study are consistent with the results obtained in the present study; the comparison is shown in Table 8 .

As it can be seen in Table 8, the percentage of students who answered either "increases" or "stays the same" remains consistent from the previous application to the two versions of the present study. This reinforces the validity of the present study and its results. The sections that follow discuss new findings that this study contributes to the field, and their possible implications. 
Table 8. Compares the results obtained in a preliminary study with the results obtained in the present. It can be seen that with different sample populations, the percentages of students who answer either "increases" or "stays the same" remains consistent

\begin{tabular}{|l|c|c|c|}
\hline & $\begin{array}{c}\text { Previous Study - Version with Electric Field } \\
\text { Lines }\end{array}$ & Present Study - V1 & Present Study - V2 \\
\hline Increases & $48 \%$ & $48 \%$ & $50 \%$ \\
\hline Stays the same & $46 \%$ & $42 \%$ & $42 \%$ \\
\hline
\end{tabular}

\section{Superposition Principle}

Besides the blocking effect created by electric field lines, the presence of charges in the diagram seems to have another effect on the application of the superposition principle, as seen in Table 3 there is a decrease of $25 \%$ on the use of the superposition principle from V1 to V2. The superposition principle refers to the contribution of electric field that each of the sources makes on the electric field at a certain point. In an electric field lines diagram, the source of electric field is present and it makes sense to apply the superposition principle; it is easy to identify each source of electric field and draw the vectors that represent the contribution that each source exerts, and do a vector sum. On the other hand, in a diagram where the source of electric field is not present, it does not make much sense to analyze the electric field of each source as if it were by itself. In this case, the student needs to recur to the diagram shown and make an interpretation of it, which is usually incorrect or incomplete; these interpretations can lead the student to identify that there are two charges creating the field, or to think about repulsion, which may lead them to a right answer (or not, as discussed later), but is not a complete interpretation of the diagram.

The correct interpretation of the electric field lines diagram would recur to the density of lines, or to the amount of lines passing through a line or a plane on that point. Alarmingly, only two students recurred vaguely to this interpretation in V2, and none of the students in V1.

\section{Repulsion}

In a previous study by Garza and Zavala (2013), it was found that students have more problems understanding the concept of electric field than electric force. There are a few possible ways of mixing the concepts of electric force and electric field that were found in the reasoning given by students. Some students talk about electric field lines as "force lines", and identify the gap between the lines as repulsion. Other students talk indifferently about electric field and electric force, or talk about point $P$ as a positive test charge that feels repulsion from the source charges.

Some students use repulsion to justify that the electric field stays the same in V1, and a similar percentage uses repulsion to state that it increases in V2. A deeper look into the full reasoning is needed to understand this phenomenon. It seems to be that when students see two charges that create an electric field they identify there is repulsion because they see that the field lines that leave the charge on the left do not reach point $\mathrm{P}$; this effect may lead them to think that the additional charge does not affect this point, as stated by student 2 on Table 6 . On the other hand, when they see an electric field represented by field lines without charges, they can easily identify that there is repulsion, which leads them to think there are two charges, and since there are two charges creating the field, then its magnitude increases, 
as stated by student 3 in Table 7. There is no complete understanding of this effect with the available information, a study focused on this effect would be needed to better analyze these results.

Furthermore, as shown in Table 3, in V1, 45\% of the students who answered that the electric field increases, based their answer on the superposition principle, while $8 \%$ based it on the concept of repulsion. Contrastingly, in V2, 20\% of the students who answered that the electric field increases, based their answer on the superposition principle, while $20 \%$ based it on repulsion. These differences suggest that the diagram with charges favors the argument of superposition, and in the diagrams without charges students need to recur to use repulsion as a tool to interpret the diagram, and this causes a decrease in the use of superposition.

\section{Special Case of Blocking Effect by Field Lines}

When analyzing the blocking effect caused by field lines, there is one case where the student is aware of the blocking effect and mentions it, but uses the superposition principle to assert that the electric field increases; this is included as student 5 in Table 6 . This reasoning is interesting for this case, but is definitely not enough for other cases.

The case of study presented in this chapter represents one of the simplest distributions of charge. Even when the student cited above identified that there is a visual blocking effect, and used the superposition principle instead, this ability will not always be enough to justify more complicated distributions. This was found in V2, where there is no source of charge present in the diagram, and the student can have difficulties finding the source of charge in more complicated distributions, making it impossible to apply the superposition principle.

To correctly interpret the electric field lines diagram, the student needs to refer to 1) the density or closeness of electric field lines to determine the magnitude of the electric field and 2) to a vector tangent to the line to determine the direction of the electric field. As mentioned previously, students do not interpret the diagram of electric field lines correctly, since they do not use these characteristics to justify their answers. If students do not interpret electric field lines correctly, then the question about whether it is worth it to teach them and to use them as a tool rises. To some extent, the diagram creates more confusion than guidance on students' comprehension of the electric field.

\section{SOLUTIONS AND RECOMMENDATIONS}

As the findings in the present study suggest, the use of electric field line diagrams carries misconceptions in the understanding of the electric field. Teaching how to draw and interpret electric field line diagrams correctly would need a large investment of time both for professors and students (Chabay \& Sherwood, 2006); and at a certain degree, it would render unfruitful, since the magnitude of the field cannot be accurately interpreted from the electric field line diagram alone (Wolf, Van Hook \& Weeks, 1996).

As supported by literature, the problems related to electric field line diagrams can be avoided by using other, more accurate representations of the field (Wolf, Van Hook \& Weeks, 1996; Chabay \& Sherwood, 2006). It should be considered to substitute electric field line diagrams by the use of vectors that represent the electric field. The vector representation is more helpful for the correct application of the superposition principle (Garza \& Zavala, 2010; Zavala, Campos \& Guisasola, 2016). By the time 


\section{A Look into Students' Interpretation of Electric Field Lines}

students start an EM course, they should be comfortable using vectors (Chabay \& Sherwood, 2006); this previous knowledge should be used as an advantage to better student's comprehension and to use teaching-learning time more wisely.

\section{FUTURE RESEARCH DIRECTIONS}

Specifically, in this topic, and as a result of the present study, there is a need to further investigate the relation of concepts made by students regarding electric field and electric force. The concept of repulsion was used by students to support both correct and incorrect answers, and at this moment, there is not full comprehension on this observed behavior.

A more general topic of interest related to the study is to analyze the effect that each representation has on the understanding of electric field, and what specific topics and models are stimulated by each. In a broader sense, professors can learn what representations of the electric field to use when teaching each topic, leading to a better quality of education and use of time.

\section{CONCLUSION}

In Electricity and Magnetism, the electric field is one of the main and most basic concepts because the study of electrostatics at the undergraduate level is focused on it, either directly or indirectly. The electric field is also an abstract concept, since it is something that cannot be directly measured, but is useful to understand interactions at a distance. The electric field has a vector nature because it has magnitude and direction, and it obeys the superposition principle due to its linearity (Serway \& Jewett, 2010). The electric field can be represented through the vector diagram and through electric field lines; these representations play a key role in the understanding of the concept of electric field because they are directly related to the conceptual models of action-at-a-distance and the field model (Furio \& Guisasola, 1998). The vector representation is more related to the model of action-at-a-distance because it considers the electric field as the force that a positive test charge would experience if it were placed at a specific position in space; the electric field line representation is more related to the field model because it implies that the source of field modifies space, and that interactions occur through the field, more explicitly that the source interacts with the field and the field interacts with the test charge.

In previous studies it was found that misinterpretations in the electric field lines diagram may result in a misapplication of the superposition principle (Zavala, Campos \& Guisasola, 2016). This is a problem because the superposition principle is one of the most powerful tools that students have when calculating electric fields exerted by known distributions of charge. After these findings, the need to further investigate students' interpretation of the electric field lines diagram was evident. The hypothesis is that the blocking effect might be caused by a visual disadvantage of the diagram when all of the elements are present. The question that this investigation tackled was: what would happen if students were shown a diagram of electric field lines omitting the charges that create it? Would it be easier to understand or would it be more complicated?

The instrument consisted of two versions of a test with open-ended questions about the electric field on a particular case. The tests were administered in Spanish in a private Mexican university. The administration of the two versions was random to undergraduate engineering students taking the course 
of Electricity and Magnetism (EM) upon completion of the topic of electric field. On every case, the students were asked to explicitly post the reasoning that led them to their answer.

The five main results found on the analysis of students' reasoning are:

1. A blocking effect caused by electric field lines is present in both versions of the test, and consistent with a previous study (Zavala, Campos \& Guisasola, 2016). This visual blocking effect is caused by the deviation that field lines have because one of the rules of electric field line diagrams is that field lines cannot cross each other (Serway \& Jewett, 2010).

2. The superposition principle may be favored by the presence of charges in the electric field lines diagram. When students are able to detect the source of electric field in the diagram, they do not need to use electric field lines to describe the electric field, and therefore they can recur to their knowledge of the superposition principle of electric fields. In the case where they do not know the distribution of charge, the application of the superposition principle is impossible; students could try to imagine the distribution or arrangement of charges for simple cases like the one presented in this study, but for more complicated cases this is not an option.

3. There is a possible relation in the decrease of the use of superposition principle when there are no charges in the diagram, with an increase in the use of repulsion in the same case. The deviation of electric field lines may lead students to think about repulsion between charges of the same sign, but this relation requires further investigation.

4. Surprisingly, in V2, in which explicit charges are not present, students did not base their explanations on the density of field lines or the separation between them, something the textbook explains and emphasizes. It is important to recall that in the electric field lines diagram, the separation between lines or density of lines is the only source of information for the relative magnitude of the electric field.

5. Finding 4 supports the claim that students do not interpret electric field lines correctly; in addition, the use of field lines may be causing more confusion than guidance in the understanding of the concept of electric field and the important underlying principles.

There are some advantages and disadvantages of using electric field lines diagrams. Some of the advantages are that they give the student a sense of the direction and the relative magnitude of the electric field in the space, and that they are visually attractive for students. Some of the disadvantages are that they fail to accurately represent the magnitude of the electric field at any point in space (Wolf, Van Hook \& Weeks, 1996), that they create blocking effects and lead to a misapplication of the superposition principle (Garza \& Zavala, 2013; Zavala, Campos \& Guisasola, 2016), and that students do not interpret electric field lines correctly, as found in the present study. These results raise the question of whether it is worth it to teach electric field lines in the basic EM course. At the undergraduate level, it might be counteractive to use several representations for the confusion that it may create on students' understanding.

\section{REFERENCES}

Barniol, P., \& Zavala, G. (2009). Investigation of Students' Preconceptions and Difficulties with the Vector Direction Concept at a Mexican University. American Institute of Physics Conference Proceedings, 1179, 85-88. 
Chabay, R., \& Sherwood, B. (2006). Restructuring the introductory Electricity and Magnetism course. American Journal of Physics, 74(4), 329-336. doi:10.1119/1.2165249

Furio, C., \& Guisasola, J. (1998). Difficulties in Learning the Concept of Electric Field. Science Education, 82(4), 511-526. doi:10.1002/(SICI)1098-237X(199807)82:4<511::AID-SCE6>3.0.CO;2-E

Garza, A., \& Zavala, G. (2010). Electric Field Concept: Effect of the Context and the Type of Questions. Physics Education Research Conference 2010. American Institute of Physics Conference Proceedings, $1289,145-148$.

Garza, A., \& Zavala, G. (2013). Contrasting Students' Understanding of Electric Field and Electric Force. Physics Education Research Conference. American Institute of Physics Conference Proceedings, 1513, 142-145. doi:10.1063/1.4789672

Gire, E., \& Price, E. (2013). Arrows as anchors: Conceptual blending and student use of electric field vector arrows. Physics Education Research Conference 2012. American Institute of Physics Conference Proceedings, 1513, 150-153. doi:10.1063/1.4789674

Maloney, D.P.(1985). Charged poles? Physics Education, 20(6),310-316. doi:10.1088/0031-9120/20/6/009

McDermott, L. C., \& Redish, E. F. (1999). Resource letter: PER-1: Physics education research. American Journal of Physics, 67(9), 755-767. doi:10.1119/1.19122

Rainson, S., \& Viennot, L. (1992). Students reasoning about the superposition of electric fields. International Journal of Science Education, 14(4), 475-487. doi:10.1080/0950069920140409

Sağlam, M., \& Millar, R. (2006). Upper High School Students Understanding of Electromagnetism. International Journal of Science Education, 28(5), 543-566. doi:10.1080/09500690500339613

Scaife, T. M., \& Heckler, A. F. (2007). The effect of field representation on student responses to magnetic force questions.Physics Education Research Conference, 951, 180-183. doi:10.1063/1.2820927

Serway, R. A., \& Jewett, J. W. (2010). Physics for Scientists and Engineers with Modern Physics (8th ed.; Vol. 2). Brooks/Cole Cengage Learning.

Sousa, R. G., Garcia, P. J., Marinho, V., \& Mouraz, A. (2013, October). Visualization of electric field lines in an engineering education context. In Engineering Education (CISPEE), 2013 1st International Conference of the Portuguese Society for (pp. 1-6). IEEE. doi:10.1109/CISPEE.2013.6701973

Törnkvist, S., Pettersson, K. A., \& Tranströmer, G. (1993). Confusion by representation: On students comprehension of the electric field concept. American Journal of Physics, 61(4), 335-338. doi:10.1119/1.17265

Wolf, A., Van Hook, S. J., \& Weeks, E. R. (1996). Electric field line diagrams don't work. American Journal of Physics, 64(6), 714-724. doi:10.1119/1.18237

Zavala, G., Alarcon, H., Henderson, C., Sabella, M., \& Hsu, L. (2008). Evaluation of instruction using the Conceptual Survey of Electricity and Magnetism in Mexico. AIP Conference Proceedings, 1064(1), 231-234. doi:10.1063/1.3021262

Zavala, G., Campos E., \& Guisasola, J. (2016). Electric field lines: Is it worth it to teach them? Manuscript in progress. 


\section{KEY TERMS AND DEFINITIONS}

Electric Field: The inherent ability of charged matter to interact with other charged objects at a distance. Electric Field Lines: A representation of the electric field, where the direction of the field is tangent to the line at any point, and its magnitude is proportional to the density of lines in the region.

Electric Force: Interactions between charged objects at a distance. There are only two types of interactions, attraction and repulsion.

Physics Education Research: Research made by Physics professors with the objective of bettering the teaching-learning process.

Representation: A sketch or diagram that aims to represent an abstract concept.

Repulsion: A type of electric force where charged objects push each other away without having direct contact.

Superposition of Electric Fields: At any given point, the electric field is equal to the vector sum of the electric field contributions that all sources exert on that point, as if they stood alone. 


\section{APPENDIX}

\section{Version 1: Problem Involving Two Charges}

1. There is a point charge $+\boldsymbol{q}$ at a distance $\boldsymbol{d}$ from a point $\boldsymbol{P}$ as shown in the figure (see Figure 6). Describe the electric field due to the point charge at $\boldsymbol{P}$.

2. Explain how you reached that description of the electric field on question 1 .

3. To the former case, we add an additional point charge $+\boldsymbol{q}$ at a distance $\boldsymbol{d}$ to the left of the original charge, as shown in the figure (see Figure 7). Is the magnitude of the electric field at point Pgreater than, less than or equal to the magnitude of the field at the same point in question 1 ?

4. Explain why the electric field increases, decreases, or stays the same in comparison to the electric field on question 1.

Figure 6. Diagram for question 1 of Version 1

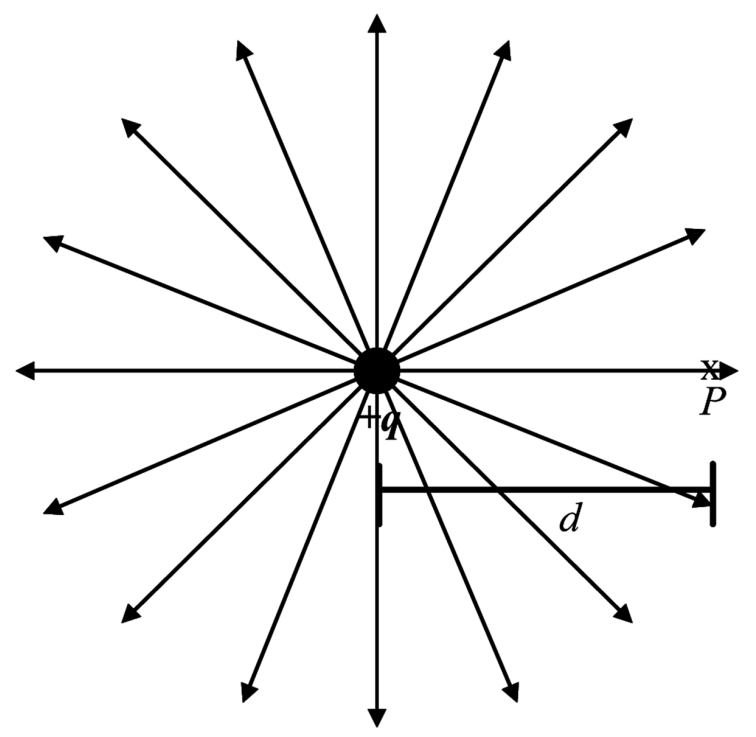

Figure 7. Diagram for question 3 of Version 1

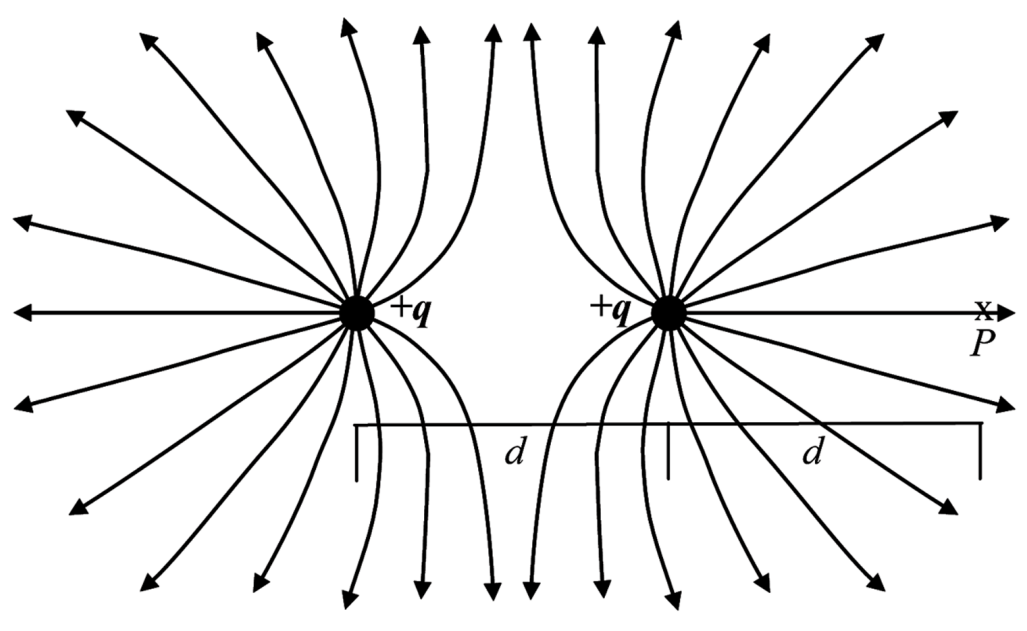




\section{Version 2: Problem Involving Two Charges}

1. There is a point charge $+\boldsymbol{q}$ at a distance $\boldsymbol{d}$ from a point $\boldsymbol{P}$ as shown in the figure (see Figure 8). Describe the electric field due to the point charge at $\boldsymbol{P}$.

2. Explain how you reached that description of the electric field on question 1.

3. To the former case, we add an additional point charge $+\boldsymbol{q}$ at a distance $\boldsymbol{d}$ to the left of the original charge, as shown in the figure (see Figure 9). Is the magnitude of the electric field at point $\boldsymbol{P}$ greater than, less than or equal to the magnitude of the field at the same point in question 1?

4. Explain why the electric field increases, decreases, or stays the same in comparison to the electric field on question 1.

Figure 8. Diagram for question 1 of Version 2

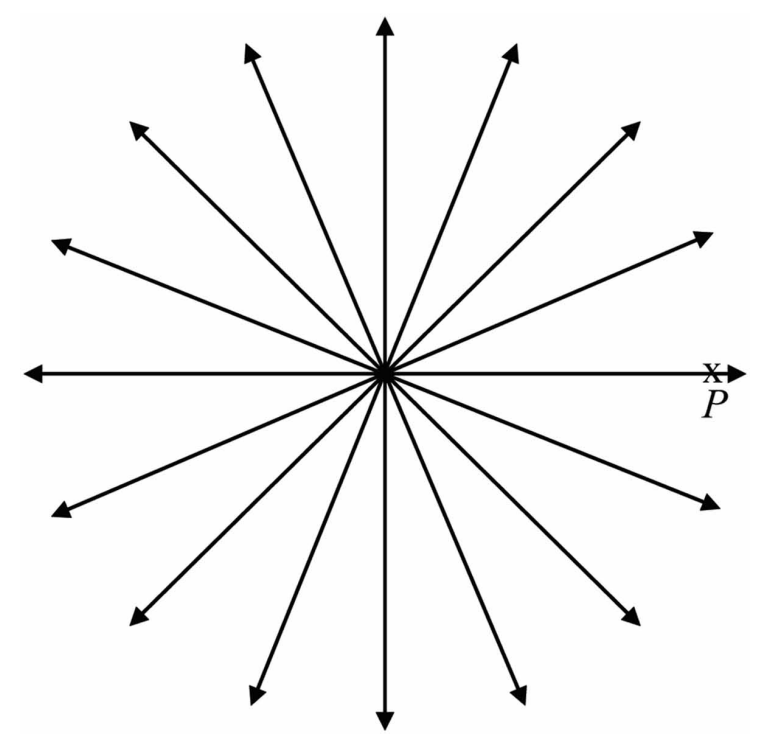

Figure 9. Diagram for question 3 of Version 2.

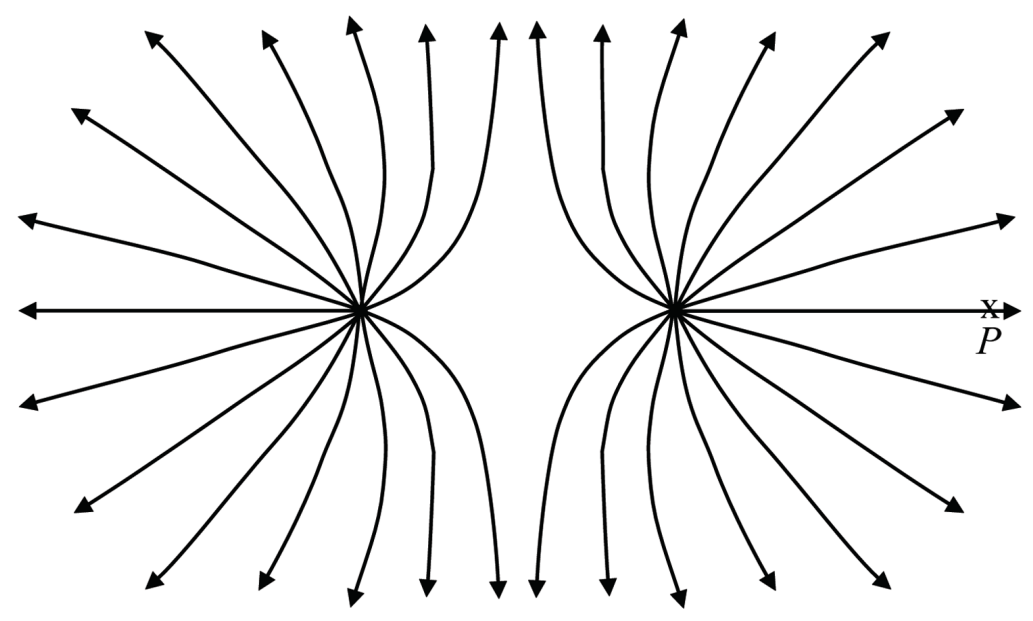

